\title{
Light coherence properties in optical fibres and visual receptors
}

\section{David James Carpenter}

The optical field on the end of an optical fibre can display varying degrees of spatial coherence. The classical analyses of the excitation of the fibre have relied on the assumptions that the coherence properties of this field can be approximated by either perfect coherence or total incoherence, and it has long been appreciated that the resulting excitations are markedly different.

It is the purpose of the work presented in this thesis to investigate the effects of differing degrees of coherence of this excitation field. The investigation has been restricted in the main to the bound or guided power of the fibre, and the prime consideration has been of current practical communications fibres.

Chapter I provides some necessary background information for the thesis and discusses the types of optical fibres to which the study is applied.

Chapter II contains a discussion of the theory of partial coherence. The history of optical coherence theory is summarised and then the formal treatment of the theory is described. In the last part of the chapter, an alternative representation of a partially coherent field by an angular spectrum of plane waves is introduced. This is of considerable importance, as it has been used extensively in the work presented here in the framework of the geometric optics description of optical fibre excitation.

The need to normalise the power launched into an optical fibre by a partially coherent source led to an investigation of the diffraction of

Received 1 June 1977. Thesis submitted to the Australian National University, April 1977. Degree approved, August 1977. Supervisors: Dr Colin Pask, Professor Allan W. Snyder. 
partially coherent light. This is contained in Chapter III. After a discussion of the classical diffraction theory and of previous studies of the diffraction of partialiy coherent light, a vector formulation is presented, which is shown to have some advantages over the previous analyses. The angular spectrum representation is then used to examine the problem, and shown to give some very informative, if qualitative, results.

The central problem of the excitation of an optical fibre by a partially coherent source is tackled in the next two chapters. In Chapter IV the excitation mechanism is discussed in terms of both mode theory and geometric optics. A general analysis for polychromatic partially coherent sources is then developed and the limiting forms of total coherence and total incoherence-are derived. A quasimonochromatic approximation is also obtained from this general result. A separate derivation of the quasimonochromatic form is then presented. These analyses are restricted to large $V$ fibres, but are independent of the fibre type.

In Chapter $V$, the quasimonochromatic analysis is applied to the step index fibre, giving detailed results for $V \leq 20$ and asymptotic expressions for $V \gg 1$ and for the two extremes of total coherence and total incoherence. A geometric optics treatment of the step index fibre is then presented, and shown to be a good approximation for $V \gtrsim 10$ and for more incoherent excitations. The last part of the chapter examines the excitation of both step and graded index fibres using a more general and detailed geometric optics approach. This approach is shown to be very powerful, and is used to examine both the bound and leaky powers associated with the fibres.

Particular examples of excitations are considered in Chapter VI. In the previous chapters, the source used was the optical field on the end of the fibre, with no reference being made to specific physical sources. In this chapter the two most important sources, the light emitting diode and semiconductor laser, are examined with reference to the results obtained in Chapter $V$, and with reference to the common approximations used. The effect of a lens to increase the coupling efficiency of the light emitting diode is also examined.

The lens excitation in Chapter VI prompted the investigation of the propagation of spatial coherence that appears in Chapter VII. The propagation of the degree of spatial coherence along an optical fibre is 
investigated first, and the phenomenon of coherence enhancement is shown to occur in this situation. The effect of the temporal coherence of the source, a reflection of the finite bandwidth of any physical source, is briefly discussed. A study of the degree of coherence in the image of a partially coherent object forms the last part of this chapter. The simple rule used in Chapter VI is initially derived from intuitive ideas, then shown to be supported by a more formal approach, and finally proved by the use of Fourier theory.

The final chapter deals with the previously excluded small $V$ fibres. These are of relevance to communications systems, in the guise of monomode fibres, and to vision research, where visual photoreceptors have been successfully modelled by small $V$ optical waveguides. The relevance of the partially coherent excitation theories developed in this thesis is discussed with reference to these two examples of small $V$ fibres. 